\title{
Pancreaticoduodenectomy with combined hepatic artery and portal vein resection after laparoscopic division of pancreaticosplenic ligament due to FOLFIRINOX-induced hepatic toxicity related secondary hypersplenism
}

\author{
Ji Su Kim ${ }^{1,4}$, Jeong Youp Park ${ }^{2,4}$, Munseok Choi ${ }^{5}$, Dong Jin Joo, \\ Ho Kyoung Hwang ${ }^{1,4}$, Woo Jung Lee ${ }^{1,4}$, and Chang Moo Kang ${ }^{1,4^{\prime}}$
${ }^{1}$ Division of Hepatobiliary and Pancreatic Surgery, ${ }^{2}$ Division of Gastroenterology, Department of Internal Medicine, ${ }^{3}$ Division of Transplantation, Department of Surgery, Yonsei University College of Medicine, ${ }^{4}$ Pancreatobiliary Cancer Center, Yonsei Cancer Center, Severance Hospital, Seoul, ${ }^{5}$ Department of Surgery, Yongin Severance, Yongin, Korea

\begin{abstract}
Pancreatic cancer is one of the dismal malignant disease in gastrointestinal tract. However, since the recent literature reporting median survival of FOLFIRINOX (leucovorin clcium, fluorouracil, irinotecan hydrochloride, oxaliplatin) chemotherapy was more than 12 months in metastatic pancreatic cancer was published, the positive attitude toward the treatment of the advanced pancreatic cancer is gradually expanded among the medical and surgical oncologists. Due to multiple combination of potent chemotherapeutic agents, potential adverse side effects should be concerned when considering FOLFIRINOX. Herein, we report a 55-year old male patient with locally advanced pancreatic cancer who successfully underwent curative resection following by laparoscopic division of pancreaticosplenic ligament due to long-term preoperative use of FOLFIRINOX related hepatic toxicity associated with secondary hypersplenism. The present case suggests the extended radical PD with combined major vascular resection following laparoscopic division of pancreaticosplenic ligament containing splenic artery and vein can improve the safety of curative resection and may expand the potential indication of pancreatic cancer in well-selected long-term use of preoperative FOLFIRINOX induced hepatic toxicity associated with secondary hypersplenism. (Ann Hepatobiliary Pancreat Surg 2021;25:307-312)
\end{abstract}

Key Words: Pancreaticoduodenectomy; FOLFIRINOX; Hypersplenism; Liver toxicity

\section{INTRODUCTION}

Is FOLFIRINOX (leucovorin calcium, fluorouracil, irinotecan hydrochloride, oxaliplatin) light to the pancreatic cancer treatment? It is known that pancreatic cancer is one of the dismal malignant disease in gastrointestinal tract. However, since the recent literature reporting median survival of FOLFIRINOX chemotherapy was more than 12 months in metastatic pancreatic cancer was published, ${ }^{1,2}$ the positive attitude toward the treatment of the advanced pancreatic cancer is gradually expanded among the medical and surgical oncologists. It was also reported that the long-term survival outcome of FOLFIRINOX was found to be extremely superior to conventional gemcitabine in postoperative adjuvant setting. ${ }^{3}$

Due to multiple combination of potent chemotherapeutic agents, potential adverse side effects should be concerned when considering FOLFIRINOX in clinical practice. ${ }^{4}$ In addition, with advance of surgical techniques for margin-negative pancreatectomy and under the rationales to support several oncologic benefits of neo-adjuvant chemotherapy in pancreatic cancer, conversion surgery following preoperative chemotherapy is actively considered in locally advanced pancreatic cancer. ${ }^{5}$

In this case, we report successful pancreaticoduodenectomy (PD) with combined vascular resection following

Received: October 6, 2020; Revised: November 17, 2020; Accepted: November 26, 2020

Corresponding author: Chang Moo Kang

Division of Hepatobiliary and Pancreatic Surgery, Department of Surgery, Yonsei University College of Medicine, 50 Yonsei-ro, Seodaemun-gu, Seoul 03722, Korea

Tel: +82-2-2228-2100, Fax: +82-2-313-8289, E-mail: cmkang@yuhs.ac

Copyright (C) 2021 by The Korean Association of Hepato-Biliary-Pancreatic Surgery

This is an Open Access article distributed under the terms of the Creative Commons Attribution Non-Commercial License (http://creativecommons.org/ icenses/by-nc/4.0) which permits unrestricted non-commercial use, distribution, and reproduction in any medium, provided the original work is properly cited. Annals of Hepato-Biliary-Pancreatic Surgery - pISSN: 2508-5778 - elSSN: 2508-5859 
laparoscopic division of pancreaticosplenic ligament in patients with FOLFIRINOX-induced hepatic toxicity associated secondary hypersplenism.

\section{CASE}

\section{Case presentation}

A 55 years old male initially visited the hospital with jaundice. He doesn't have any other clinical disease and past history. Computed tomography(CT) imaging identified a tumor invaded more than 180 degrees in Common hepatic artery (CHA), Gastroduodenal artery (GDA), Portal vein (PV) (Fig. 1A). He diagnosed locally advanced pancreatic head cancer with major vascular invasion. After endoscopic retrograde biliary drainage (ERBD), he started neo-adjuvant chemotherapy with FOLFIRINOX full dose
(147.9 $\mathrm{mg} / \mathrm{m}^{2}$ of oxaliplatin, $348 \mathrm{mg} / \mathrm{m}^{2}$ of leucovorin, $313.2 \mathrm{mg} / \mathrm{m}^{2}$ of irinotecan, and a total of $2784 \mathrm{mg} / \mathrm{m}^{2}$ of fluorouracil) in February 2017. When he received 11th FOLFIRINOX, he developed neutropenia and thrombocytopenia, requiring dose reduction to $75 \%$.

After finished 12th FOLFIRINOX, the tumor was stable and responded to neo-adjuvant chemotherapy. Even though still invaded CHA and PV, tumor size was decreased to $2 \mathrm{~cm}$ from $3 \mathrm{~cm}$. It was still in unresectable status, so he was decided to take concurrent chemo-radiation therapy (CCRT) with Xeloda in September 2017. During CCRT, his neutropenia and thrombocytopenia got worse. In December 2017, he started chemotherapy with 13th FOLFIRINOX, only $25 \%$ dose used. From 15th FOLFIRINOX, 50\% dose was used. In March 2019, after finishing all 32th FOLFIRINOX, follow up CT scan
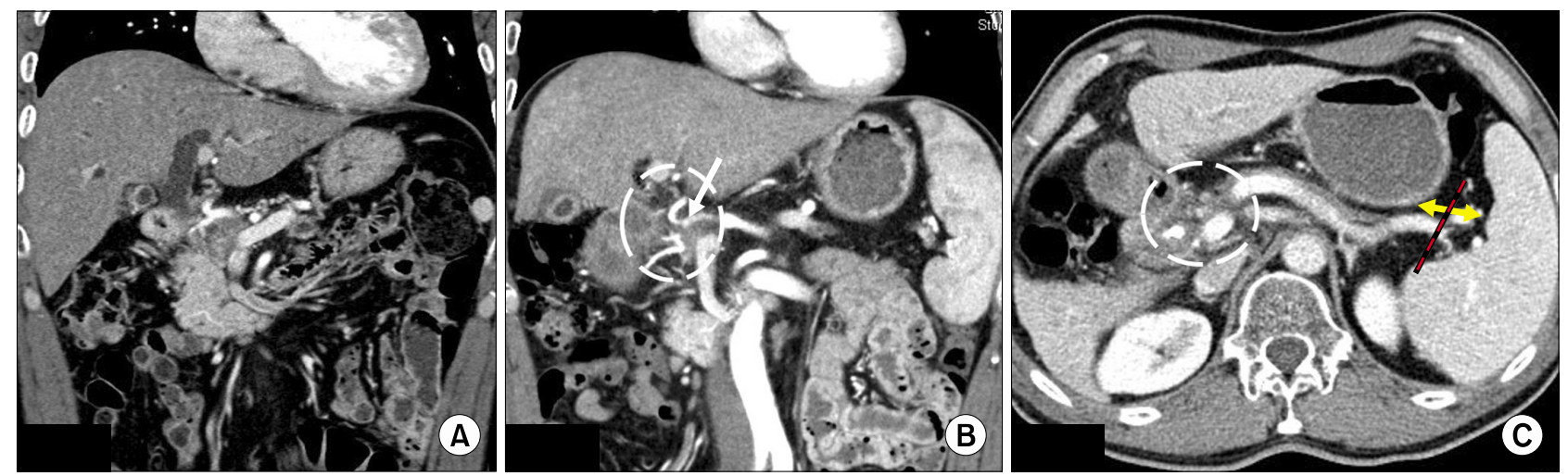

Fig. 1. Preoperative CT scan. Invasion of pancreatic head cancer around SMV-SV-PV confluence and CHA was noted at the time of diagnosis (A). Soft tissue infiltration was still noted without progression after neo-adjuvant chemotherapy (B). White Dotted circle indicates tumor area and thick long white arrow indicates cancer infiltrating around common hepatic artery (B, C). Note atrophic change of distal part of the pancreas makes enough space between tip of the pancreas and splenic hilum (yellow arrow). Potential division line of pancreaticosplenic ligament was noted (red dotted line, C). CT, computed tomography; $\mathrm{SMV}$, superior mesenteric vein; SV, splenic vein; PV, portal vein; CHA, common hepatic artery.
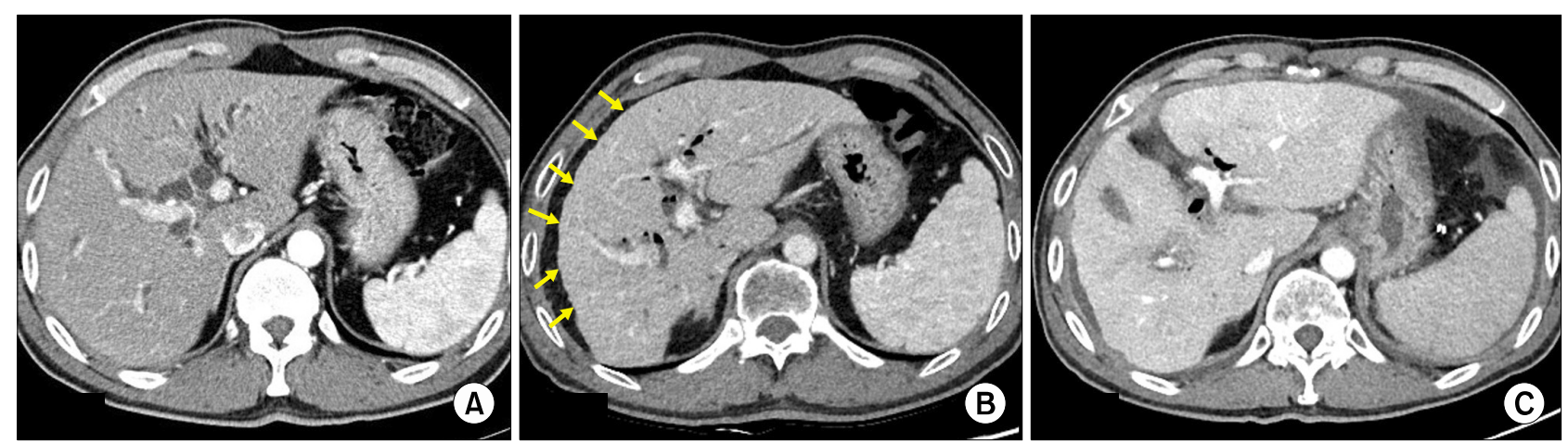

Fig. 2. Liver volume at initial CT (A), preoperative (B) and the postoperative (POD\#7) $C T$ (C). (A=1488.61 $\mathrm{cm}^{3}, B=1077.97$ $\mathrm{cm}^{3}, \mathrm{C}=1294.85 \mathrm{~cm}^{3}$ ) Note liver atrophy (yellow arrow) after long-term neoadjuvant chemotherapy with relatively enlarge spleen. $\mathrm{CT}$, computed tomography; POD, postoperative days. 
showed no interval change of primary pancreatic cancer, with CHA, and PV invasion (Fig. 1B). Liver atrophy worsened and total liver volume decreased to $1077.97 \mathrm{~cm}^{3}$ from $1488.61 \mathrm{~cm}^{3}$ (Fig. 2A, B). Thrombocytopenia $(79,000$ ul) was still noted (Fig. 3), which was thought to be due to long-term use of FOLFIRINOX-induced liver toxicity related portal hypertension with secondary hypersplenism.

\section{Operation}

The Pylorus preserving pancreaticoduodenectomy (PPPD) with PV, CHA segmental resection was planned for this patient. Initially, diagnostic laparoscopy was performed to check occult metastasis. No systemic metastasis or peritoneal seeding was noted. In order to compensate potential thrombocytopenia, pancreaticosplenic ligament containing splenic artery and vein resected near the spleen hilum using an Endo-GIA stapler (Figs. 1C and 4A, B). Then laparotomy was performed to proceed PPPD with combined vascular resections. Superior mesenteric vein (SMV)-PV end to end anastomosis, and $\mathrm{CHA}^{-}$-Proper hepatic artery (PHA) end to end anastomosis were simultaneously done (Fig. 4C, D).

\section{Postoperative outcome and recovery}

The total operation duration was 641 minutes and the estimated intraoperative blood loss was $1850 \mathrm{cc}$. He received postoperative treatment in an intensive care unit for intensive care and surveillance after surgery. The two Pack of Red Blood Cells (RBC) and Fresh Frozen Plasmas (FFP) were transfused immediately. At the postoperative 3rd days, transferred to the general ward after his vital signs were stable. He recovered routinely without any specific problem except for wound seroma. On 7th post- operative days, there were no specific finding in abdomen on routine CT. Liver recovered volume from $1077.97 \mathrm{~cm}^{3}$ to $1294.86 \mathrm{~cm}^{3}$ (Fig. 2B, C). After improved wound, He discharged hospital at the 15 days after surgery. At the discharge day, blood platelet count was found to be 334,000 ul (Fig. 3).

\section{Pathological examination}

The final histopathological result was reported as a ductal adenocarcinoma of moderate differentiation after chemotherapy. All resection margins were reported as free from carcinoma. There was no metastasis to the 8 retrieved lymph nodes. He is on follow-up for 7 months without adjuvant chemotherapy.

\section{DISCUSSION}

In this patient, the patient was initially regarded as locally advanced pancreatic cancer due to pancreatic head cancer invading to PV and CHA. Subsequent long-term use of FOLFIRINOX-based chemotherapy could prevent cancer progressing. However, liver cirrhosis-like liver contour with small ascites and secondary thrombocytopenia due to hypersplenism need to be considered in planning surgical tactics in treating this patient. The surgical procedure needed to be determined, considering curative resection, patient's safety and long-term scenario of potentially worsening liver function after pancreatectomy.

FOLFIRINOX is frequently used in the neo-adjuvant setting to downsize borderline resectable or locally advanced pancreatic cancer patients because it improves the overall survival rate and slows progression of the cancer. ${ }^{6,7}$ However, there are strong correlation between oxaliplatin

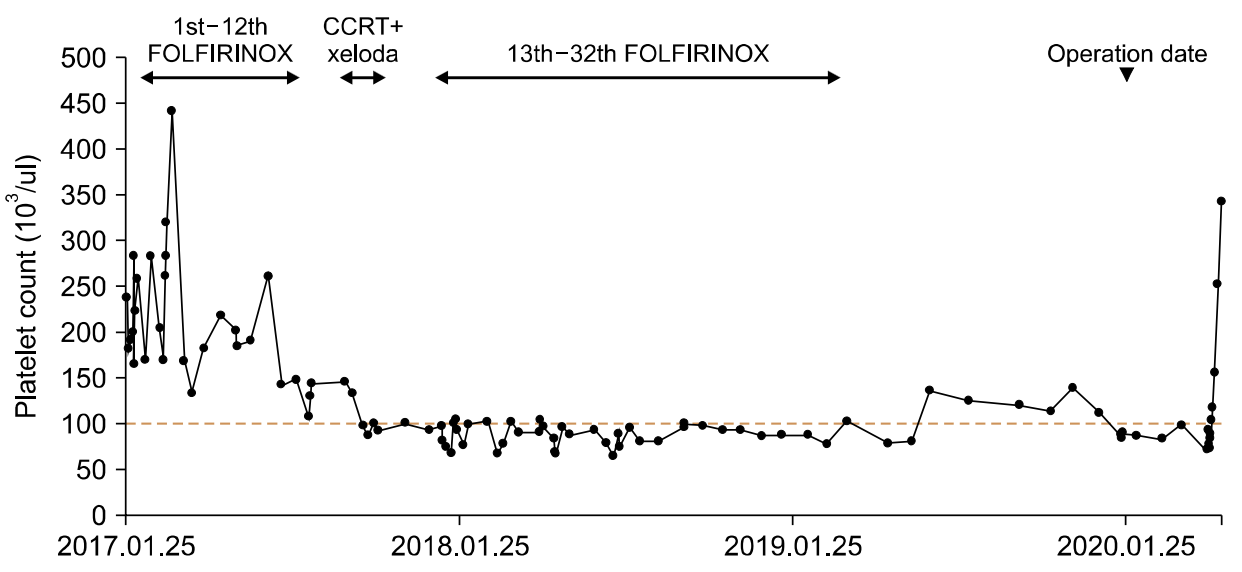

Fig. 3. Chronological change of blood platelet count according to pancreatic cancer treatment. FOLFIRINOX, leucovorin calcium, fluorouracil, irinotecan hydrochloride, oxaliplatin; CCRT, concurrent chemoradiation therapy. 

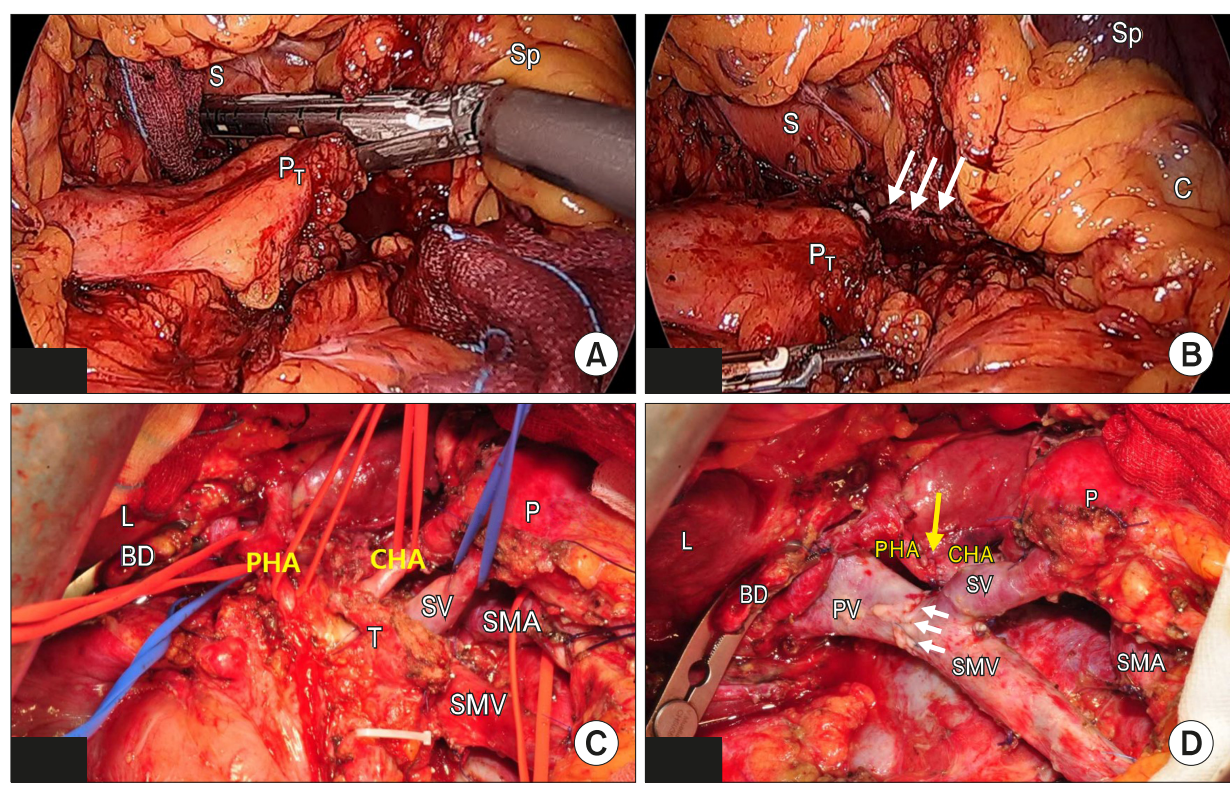

Fig. 4. Operative finding: Laparoscopic division of pancreaticosplenic ligament containing both splenic artery and vein near spleen hilum using Endo-GIA stapler (stapler line-white long arrow). (A, B) Tumor invaded CHA and SMV (C), PHA-CHA (yellow long arrow) and PV-SMV "(white short arrow)", end to end anastomosis (D). Endo-GIA, endoscopic gastrointestinal anastomosis; S, Stomach; Sp, spleen; PT, pancreas tail; $\mathrm{C}$, colon; $\mathrm{BD}$, bile duct; PHA, proper hepatic artery; CHA, common hepatic artery; $\mathrm{P}$, remnant pancreas; SV, splenic vein; T, tumor; SMA, superior mesenteric artery; SMV, superior mesenteric vein. and sinusoidal obstruction syndrome, ${ }^{8,9}$ which is well known one of the causes of portal hypertension. In this patients, the patient's initial liver contour and blood platelet count were normal. After long-term 32th cycle of FOLFIRINOX, sustained thrombocytopenia and atrophied liver shown on preoperative CT scan were suggesting repeated cycles of FOLFORINOX might result in sinusoidal obstruction syndrome and secondary hypersplenism due to subsequent portal hypertension.

Considering potential adverse impact of preoperative long-term use of FOLFIRINOX, PD with combined segmental resection of CHA and PV after laparoscopic division of both splenic artery and vein near the splenic hilum was planned for this patients. This strategy is thought to be able to preserve the spleen, resolve the secondary thrombocytopenia and temporally prevent future potential perigastric varix by disconnecting all venous communication from portal venous system (During surgical procedure of $\mathrm{PD}$, right gastric vein, right gastroepiploric vein, left gastric vein, and distal splenic vein were all divided), expecting improvement of patient' liver function and safety of the extended surgical procedure, as well. As result, it was observed that preoperative thrombocytopenia, liver volume, and liver function were all improved postoperatively in the present case.

There are several studies showing combined splenectomy could improve liver function in patients with liver cirrhosis. ${ }^{10-12}$ However, spleen is one of the largest im- munologic organ in human body, and splenectomy can be associated with infection-related postoperative complications $^{13}$ and severe sepsis. ${ }^{14}$ In addition, cancer patient, potential role of spleen as anticancer effect is concerned. Especially, Hwang, et al. ${ }^{15}$ recently observed multiple peritoneal seeding nodules of recurrent pancreatic cancer in splenectomized mouse (25\%), suggesting decreased activity of NK cell after splenectomy as possible mechanism of multiple peritoneal seeding in splenectomy group. Therefore, instead of concomitant splenectomy, the present approach, just division of both splenic artery and vein near the splenic hilum, is thought to be the best option when planning for curative intent PD in pancreatic cancer patients with portal hypertension with secondary hypersplenism.

Initial laparoscopic approach was considered in this patient; First, unexpected detection of peritoneal seeding and hepatic metastasis is not uncommon in patients with advanced pancreatic cancer. ${ }^{16,17}$ Second, it is thought that laparoscopic division of pancreaticosplenic ligament to control both splenic artery and vein is one of the common procedures for laparoscopic splenic vessels-sacrificing spleenpreserving distal pancreatectomy, so called Warshaw's procedure. ${ }^{18,19}$ Lastly, obtaining operative field and surgical manipulation around splenic hilum is thought to be much easier under the laparoscopic view than open approach.

PD with combined hepatic artery and PV resection fol- 
lowing neo-adjuvant chemotherapy is currently feasible surgical procedure in well-selected borderline and locally advanced pancreatic cancer. ${ }^{20-22}$ Not to mention of advance in surgical technique for curative resection and postoperative management, introduction of potent chemotherapeutic agents and improving patients general condition will increase long-term oncologic outcome of resected pancreatic cancer in near future.

The present case suggests the extended radical PD with combined major vascular resection following laparoscopic division of pancreaticosplenic ligament containing splenic artery and vein can improve the safety of curative resection and may expand the potential indication of pancreatic cancer in well-selected long-term use of preoperative FOLFIRINOX induced hepatic toxicity associated with secondary hypersplenism. The more active application of FOLFIRINOX in locally advanced pancreatic cancer, the more cases like the present report are highly expected to be encountered in clinical practice. The long-term effect of this surgical approach is still questioned. Further experiences are mandatory.

\section{CONFLICT OF INTEREST}

The authors have no conflicts of interest to disclose.

\section{ORCID}

Ji Su Kim: https://orcid.org/0000-0002-9501-9665

Jeong Youp Park: https://orcid.org/0000-0003-0110-8606

Munseok Choi: https://orcid.org/0000-0002-9844-4747

Dong Jin Joo: https://orcid.org/0000-0001-8405-1531

Ho Kyoung Hwang: https://orcid.org/0000-0003-4064-7776

Woo Jung Lee: https://orcid.org/0000-0001-9273-261X

Chang Moo Kang: https://orcid.org/0000-0002-5382-4658

\section{AUTHOR CONTRIBUTIONS}

Conceptualization: JSK, CMK. Data curation: JSK, MC, HKH, WJL, CMK. Formal analysis: JSK, JYP, MC, DJJ, HKH, WJL, CMK. Methodology: JSK, CMK. Project administration: JSK, CMK. Visualization: JSK. Writing - original draft: JSK, CMK. Writing - review \& editing: JSK, CMK.

\section{REFERENCES}

1. Conroy T, Desseigne F, Ychou M, Bouché O, Guimbaud R, Bécouarn Y, et al. FOLFIRINOX versus gemcitabine for metastatic pancreatic cancer. N Engl J Med 2011;364:1817-1825.

2. Sohal DP, Mangu PB, Khorana AA, Shah MA, Philip PA, O'Reilly EM, et al. Metastatic pancreatic cancer: American Society of Clinical Oncology clinical practice guideline. J Clin Oncol 2016;34:2784-2796.

3. Conroy T, Hammel P, Hebbar M, Ben Abdelghani M, Wei AC, Raoul JL, et al. FOLFIRINOX or gemcitabine as adjuvant therapy for pancreatic cancer. N Engl J Med 2018;379:2395-2406.

4. Stein SM, James ES, Deng Y, Cong X, Kortmansky JS, Li J, et al. Final analysis of a phase II study of modified FOLFIRINOX in locally advanced and metastatic pancreatic cancer. $\mathrm{Br} \mathrm{J}$ Cancer 2016;114:737-743.

5. Clancy TE. Surgery for pancreatic cancer. Hematol Oncol Clin North Am 2015;29:701-716.

6. Janssen QP, Buettner S, Suker M, Beumer BR, Addeo P, Bachellier $P$, et al. Neoadjuvant FOLFIRINOX in patients with borderline resectable pancreatic cancer: a systematic review and patient-level meta-analysis. J Natl Cancer Inst 2019;111:782-794.

7. Boone BA, Steve J, Krasinskas AM, Zureikat AH, Lembersky BC, Gibson MK, et al. Outcomes with FOLFIRINOX for borderline resectable and locally unresectable pancreatic cancer. J Surg Oncol 2013;108:236-241.

8. Seo AN, Kim H. Sinusoidal obstruction syndrome after oxaliplatin-based chemotherapy. Clin Mol Hepatol 2014;20:81-84.

9. El Chediak A, Haydar AA, Hakim A, Massih SA, Hilal L, Mukherji D, et al. Increase in spleen volume as a predictor of oxaliplatin toxicity. Ther Clin Risk Manag 2018;14:653-657.

10. Pei Y, Chai S, Zhang Y, Zhang Z, Chen X, Zhang W. Benefits of splenectomy and curative treatments for patients with hepatocellular carcinoma and portal hypertension: a retrospective study. J Gastrointest Surg 2019;23:2151-2162.

11. Wang Q, Dang T, Meng X, Li K, Ren W, Ma X, et al. Is concomitant splenectomy necessary in radical gastric cancer surgery? A systematic review and meta-analysis. Asia Pac J Clin Oncol 2019; 15:e28-e35.

12. Yamamoto N, Okano K, Oshima M, Akamoto S, Fujiwara M, Tani J, et al. Laparoscopic splenectomy for patients with liver cirrhosis: improvement of liver function in patients with ChildPugh class B. Surgery 2015;158:1538-1544.

13. Shoup M, Brennan MF, McWhite K, Leung DH, Klimstra D, Conlon KC. The value of splenic preservation with distal pancreatectomy. Arch Surg 2002;137:164-168.

14. Dalla Bona E, Beltrame V, Liessi F, Sperti C. Fatal pneumococcal sepsis eleven years after distal pancreatectomy with splenectomy for pancreatic cancer. JOP 2012;13:693-695.

15. Hwang HK, Kang CM, Lee SH, Murakami T, Kiyuna T, Kim $\mathrm{SH}$, et al. Fluorescence-guided surgery with splenic preservation prevents tumor recurrence in an orthotopic nude-mouse model of human pancreatic cancer. Anticancer Res 2018;38:665-670.

16. Stefanidis D, Grove KD, Schwesinger WH, Thomas CR Jr. The current role of staging laparoscopy for adenocarcinoma of the pancreas: a review. Ann Oncol 2006;17:189-199.

17. Pandit S, Samant H, Kohli K, Shokouh-Amiri HM, Wellman G, Zibari GB. Incidental liver metastasis in pancreatic adenocarcinoma. J Surg Case Rep 2019;2019:rjz084.

18. Wang L, Wu D, Cheng YG, Xu JW, Chu HB, Zhang GY, et al. Warshaw technique in laparoscopic spleen-preserving distal pancreatectomy: surgical strategy and late outcomes of splenic preservation. Biomed Res Int 2019;2019:4074369. 
19. Suzumura K, Hatano E, Okada T, Fujimoto J. Perioperative and long-term outcome of the Warshaw technique in laparoscopic spleen-preserving distal pancreatectomy. Surg Laparosc Endosc Percutan Tech 2017;27:474-478.

20. Kawaguchi K, Motoi F, Yokoyama S, Takemura S, Doi T, Nozawa Y, et al. [A case of curatively resected locally advanced pancreatic cancer with combined resection of the portal vein and common hepatic artery after neoadjuvant chemotherapy]. Gan To
Kagaku Ryoho 2013;40:1875-1877. Japanese.

21. Delpero JR, Sauvanet A. Vascular resection for pancreatic cancer: 2019 French recommendations based on a literature review from 2008 to 6-2019. Front Oncol 2020;10:40.

22. Oba A, Bao QR, Barnett CC, Al-Musawi MH, Croce C, Schulick $\mathrm{RD}$, et al. Vascular resections for pancreatic ductal adenocarcinoma: vascular resections for PDAC. Scand J Surg 2020;109:1828. 